\title{
Voronoi Diagrams for Oriented Spheres
}

\author{
F. Aurenhammer \\ J. Wallner \\ University of Technology \\ Graz, Austria \\ auren@igi.tugraz.at \\ j.wallner@tugraz.at
}

\author{
M. Peternell \\ H. Pottmann \\ University of Technology \\ Vienna, Austria \\ peternell@geometrie.tuwien.ac.at \\ pottmann@geometrie.tuwien.ac.at
}

\begin{abstract}
We consider finite sets of oriented spheres in $R^{k-1}$ and, by interpreting such spheres as points in $R^{k}$, study the Voronoi diagrams they induce for several variants of distance between spheres. We give bounds on the combinatorial complexity of these diagrams in $R^{2}$ and $R^{3}$ and derive properties useful for constructing them. Our results are motivated by applications to special relativity theory.
\end{abstract}

\section{Introduction}

Consider the general quadratic-form distance $Q$ for two points $p, q \in R^{k}$, given by

$$
Q(p, q)=(q-p)^{T} \cdot M \cdot(q-p),
$$

where $M$ is a nonsingular $k \times k$ matrix. We may assume that, without loss of generality, $M$ is symmetric. ${ }^{1}$ Voronoi diagrams for this kind of distance found some attention in computational geometry $[8,5,6,2]$, in particular, for special Jordan matrices $M=\operatorname{diag}\left(a_{i}\right)_{a_{i} \in\{1,-1\}}$. For each pair of points, their separator (locus of equal distance) is a hyperplane; such diagrams are sometimes called affine diagrams [2,4]. Clearly, the cases $M=I$ and $M=-I$ give the classical Voronoi diagram and its farthest-point variant, respectively. For $k=2$ and $M=\left(\begin{array}{ll}0 & 1 \\ 1 & 0\end{array}\right)$, the distance $Q(p, q)$ describes the area of the axis-parallel rectangle with diagonal $p q$. This diagram proved useful for finding the largest empty axis-parallel rectangle among a finite set of points [5]. A general result in [2] shows that all diagrams obtainable by $Q$ are power diagrams [1] for a suitable set of spheres.

In this note, we are interested in the quasi-euclidean distance, $d$, generated by the matrix $M=\operatorname{diag}(1, \ldots, 1,-1)$.

\footnotetext{
${ }^{1}$ We have $Q(p, q)=\frac{1}{2}(q-p) \cdot\left(M+M^{T}\right) \cdot(q-p)$.
}

Our main motivation comes from special relativity theory, see e.g. $[3,10]$, where one describes events as points in the quasi-euclidean space whose 'metric' is governed by the matrix $M$ and whose isometric mappings are the Lorentz transformations. The line spanned by two events $p$ and $q$ can be time-like, or space-like, or light-like - these cases correspond to $d(p, q)<0, d(p, q)>0$, and $d(p, q)=0$, respectively. The value of $d(p, q)$ is a Lorentz invariant: If positive, it has an interpretation as square of distance, and if negative, then the square root of its absolute value is the proper time experienced by a particle whose life line is the line segment $p q$. See Figure 1. The distance $d$ from event $p$ is positive in the shaded area, zero at the two 'light' lines, and negative in the blank area.

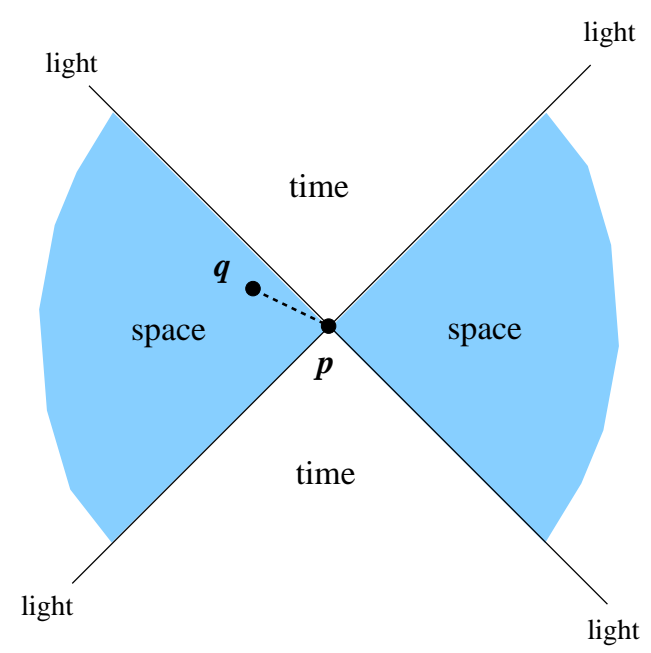

Figure 1. Time-space cones of a particle

For any finite collection of events we can now ask questions like the following: Can we decompose space into natural neighbourhood regions, and can we classify the single events as belonging to certain neighbourhood clusters? Does it make sense to distinguish strictly between time-like 


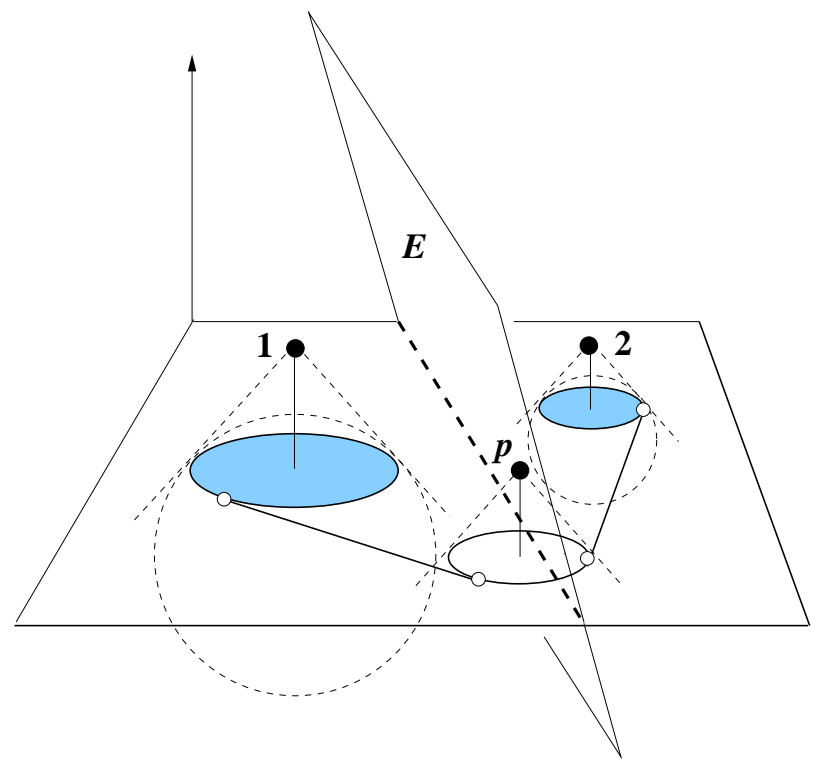

Figure 2. Separator of two oriented circles

and space-like difference vectors when doing distance computations? The present paper studies several types of cell decomposition of space, together with illustrations in $2 \mathrm{D}$, which are related to these questions. We consider decomposition into cells defined by the nearest neighbour property, but due to the nonpositivity of the function $d$, the Voronoi diagrams generated by $|d|, \max \{d, 0\}$, and related functions differ from the Voronoi diagram generated by $d$ itself.

Let us mention that the rectangle area related distance for the matrix $M=\left(\begin{array}{ll}0 & 1 \\ 1 & 0\end{array}\right)$ considered above transforms to the two-dimensional instance of $d, M=\operatorname{diag}(1,-1)$, by a rotation of $\frac{\pi}{4}$.

\section{Standard case}

We first discuss the Voronoi diagram induced by the quasi-euclidean distance $d$, without any restriction on its sign. In the physical context, this means treating life time of a particle as a negative distance in space.

We use an interpretation of $d$ based on the following simple but useful property: If the $k^{t h}$ coordinates of two points $p, q \in R^{k}$ are interpreted as 'radii', and their first $k-1$ coordinates specify 'centers', then $d(p, q)$ expresses the squared tangent length between the respective two spheres in $R^{k-1}$. That is, the space of (oriented) spheres in $R^{k-1}$ is accordingly divided by the resulting diagram in $R^{k}$.

To illustrate this fact, Figure 2 displays two circles $\sigma_{1}$ and $\sigma_{2}$ (shown as shaded disks) in the $x y$-plane, lying below their corresponding two points in $R^{3}$. Their separator with respect to the quasi-euclidean distance $d$ is a plane, $E$, that intersects the $x y$-plane in the power line of the circles $\sigma_{1}$ and $\sigma_{2}$. In fact, $E$ is the power plane of the two 3-dimensional spheres $S_{1}$ and $S_{2}$, where $S_{i}$ intersects the $x y$-plane in $\sigma_{i}$ at an angle of $\frac{\pi}{4}$, for $i=1,2$. This can be shown by simple analytic calculations. Let us call $S_{i}$ the principal sphere of $\sigma_{i}$. Note that the center of $S_{i}$ is the reflection through the $x y$-plane of the point in $R^{3}$ that corresponds to $\sigma_{i}$. Each point $p \in E$ corresponds to a circle in the $x y$-plane with tangents of equal length to $\sigma_{1}$ and $\sigma_{2}$.

Let now $\left\{\sigma_{1}, \ldots, \sigma_{n}\right\}$ be a given set of oriented spheres in $R^{k-1}$, treated as points in $R^{k}$ in the following. By the discussion above, the diagram in question is the power diagram of the corresponding $n$ principal spheres. Separators are, of course, hyperplanes in $R^{k}$, and it is easy to see that each separator indeed separates its defining spheres $\sigma_{i}, \sigma_{j}$. However, a sphere need not be contained in its halfspace of smaller distance. For each sphere $\sigma_{i}$, its region $r e g\left(\sigma_{i}\right)$ represents the set of spheres showing smallest squared tangent length to $\sigma_{i}$ (which might be negative).

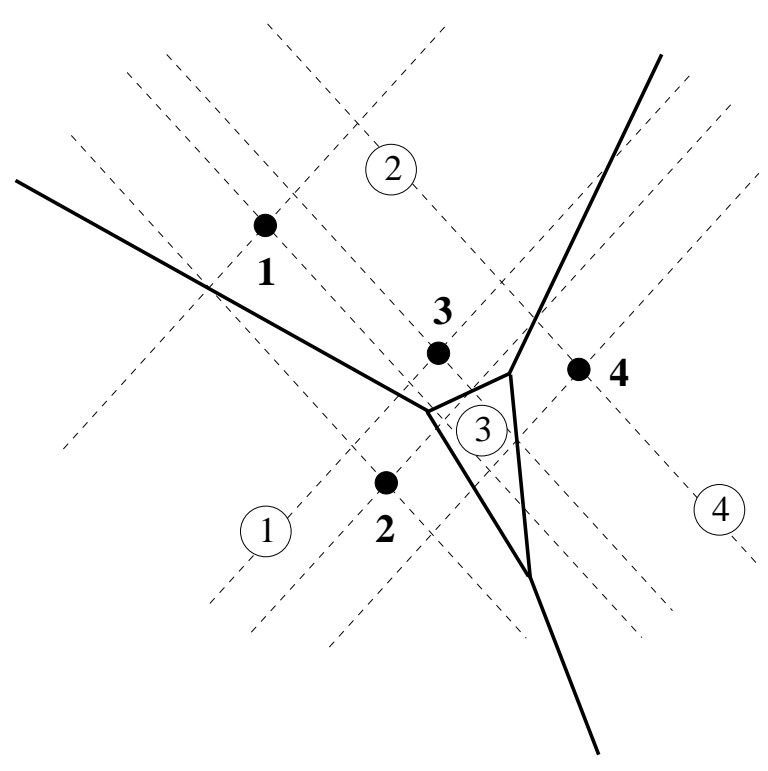

Figure 3. Standard distance

The set of all spheres at distance zero from $\sigma_{i}$ is a vertical double-cone, cone $\left(\sigma_{i}\right)$, with apex $\sigma_{i}$ and aperture angle of $\frac{\pi}{2}$. See Figure 1, where the cone apex is labeled by $p$. By slight abuse of notation, we will call the interior of cone $\left(\sigma_{i}\right)$ the part of $R^{k}$ where the distance to $\sigma_{i}$ is negative. (This corresponds to the blank area in Figure 1). The regions of the diagram are connected, as being convex polyhedra, but $\sigma_{i} \in \operatorname{reg}\left(\sigma_{i}\right)$ does not hold, in general. More precisely, we have $\sigma_{i} \notin \operatorname{reg}\left(\sigma_{i}\right)$ if and only if there exists some sphere $\sigma_{j}$ with $d\left(\sigma_{i}, \sigma_{j}\right)<d\left(\sigma_{i}, \sigma_{i}\right)=0$, that is, with $\sigma_{j}$ contained in the interior of cone $\left(\sigma_{i}\right)$. Equivalently, 
$\sigma_{i} \in \operatorname{reg}\left(\sigma_{i}\right)$ if and only if $\left\{\sigma_{1}, \ldots, \sigma_{n}\right\} \backslash\left\{\sigma_{i}\right\}$ is exterior to cone $\left(\sigma_{i}\right)$.

Figure 3 gives an example. Encirculated numbers indicate affiliation of regions to spheres. For each sphere, its double-cone is drawn in dashed lines. Sphere $\sigma_{4}$ is the only sphere fulfilling the last criterion above, thus only $\sigma_{4}$ is contained in its own region.

It can be shown that $\operatorname{reg}\left(\sigma_{i}\right)$ is unbounded if and only if $\sigma_{i}$ lies on the boundary of the convex hull of (the points) $\sigma_{1}, \ldots, \sigma_{n}$ : Let $c_{i}$ denote the center of the principal sphere $S_{i}$ for $\sigma_{i}$; cf. Figure 2. As $c_{i}$ is the reflection of $\sigma_{i}$ through the hyperplane $x_{k}=0$ of $R^{k}$, center $c_{i}$ is extreme in $c_{1}, \ldots, c_{n}$ if and only if $\sigma_{i}$ is extreme in $\sigma_{1}, \ldots, \sigma_{n}$. Extremality of centers, however, is well known to characterize unboundedness of regions in the respective power diagram. Note that $\operatorname{reg}\left(\sigma_{i}\right)=\emptyset$ is possible for non-extreme $\sigma_{i}$. The diagram (as being a power diagram) can be computed in $O(n \log n)$ time and $O(n)$ space in $R^{2}$, and in $O\left(n^{2}\right)$ time and space in $R^{3}$; see $[1,8]$. Both results are asymptotically optimal in the worst case.

\section{Variant 1}

A different behaviour is exhibited by a variant of the quasi-euclidean distance. We let

$$
D(p, q)=|d(p, q)| .
$$

Now each sphere $\sigma_{i}$ gets assigned the region of all spheres that show squared tangent lengths of smallest absolute value to $\sigma_{i}$. In physical terms, life time of a particle is just regarded as distance in space.

Two types of separators arise, namely, for the equation $d(x, p)=d(x, q)$, which gives the power hyperplanes from before, as well as for the equation $d(x, p)=-d(x, q)$, which gives hyperboloids with midpoint $\frac{p+q}{2}$. As $D(p, q) \geq 0$, the double-cones of distance zero get completely swallowed by regions; more precisely, $\operatorname{cone}\left(\sigma_{i}\right) \subset \operatorname{reg}\left(\sigma_{i}\right)$ holds for all input spheres $\sigma_{i}$. In particular, we have $\sigma_{i} \in \operatorname{reg}\left(\sigma_{i}\right)$. Figure 4 gives an illustration.

The resulting Voronoi diagram has a size of $\Theta\left(n^{2}\right)$ in $R^{2}$, because the edges of the line arrangement induced by the $n$ cones are in bijection with the interior-connected parts of the diagram regions. An exception are the $O(n)$ line arrangement edges incident to some $\sigma_{i}$. Thus the combinatorial structure of the diagram is essentially determined by the line arrangement above. More precisely, each cell $Z$ of this arrangement $(Z$ is a rectangle or an even simpler, then unbounded, polygon) is divided among at most four spheres, one for each edge of $Z$. The subdiagram within $Z$ thus is of constant size and can be computed in a straightforward way. This leads to an $\Theta\left(n^{2}\right)$ construction algorithm in $R^{2}$,

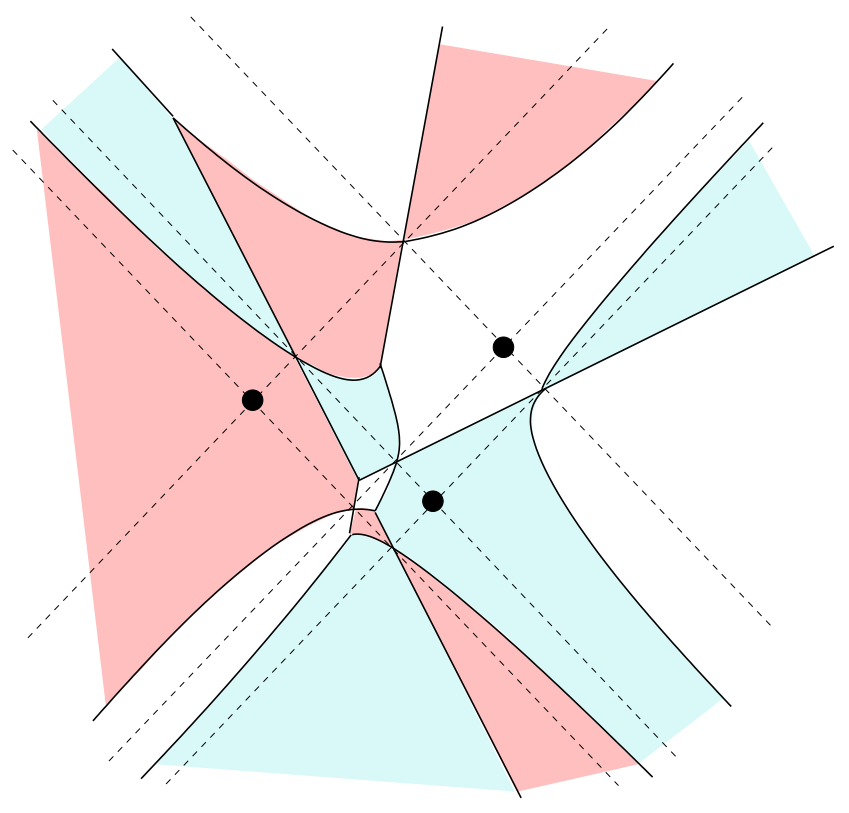

Figure 4. Distance $D$

because the number of cells is $\Theta\left(n^{2}\right)$, and all these cells can easily be constructed in $\Theta\left(n^{2}\right)$ time. Note that, as the diagram edges form a planar graph in $R^{2}$, each single region is bounded by $\Theta(n)$ edges.

By the afore-mentioned correspondence between pieces of the double-cone arrangement and diagram regions, we get a lower bound of $\Omega\left(n^{3}\right)$ for the size of the diagram in $R^{3}$. The (parts of) regions of the diagram within a fixed arrangement cell will not be constantly many, in general; their number is at most the number of facets of such a cell. However, the overall number of facets of the double-cone arrangement is $\Theta\left(n^{3}\right)$, and we conclude an asymptotically tight upper bound of $O\left(n^{3}\right)$ for the number of regions in the Voronoi diagram for distance $D$ in $R^{3}$.

\section{Variant 2}

We now restrict attention to positive tangent lengths. (In a symmetric manner, restriction to negative tangent lengths could be considered.) As one possibility, this leads us to define

$$
\Delta_{\infty}(p, q)= \begin{cases}d(p, q) & \text { if } \geq 0 \\ \infty & \text { otherwise. }\end{cases}
$$

In the resulting Voronoi diagram, spheres to which squared tangent lengths are negative are, sloppily speaking, out of the game. That is, life time of particles is not taken into account in this 'space driven' variant. More precisely, for each sphere $\sigma_{i}$, its region $\operatorname{reg}\left(\sigma_{i}\right)$ is contained in the exterior of cone $\left(\sigma_{i}\right)$. On the other hand, cone $\left(\sigma_{i}\right)$ has to 


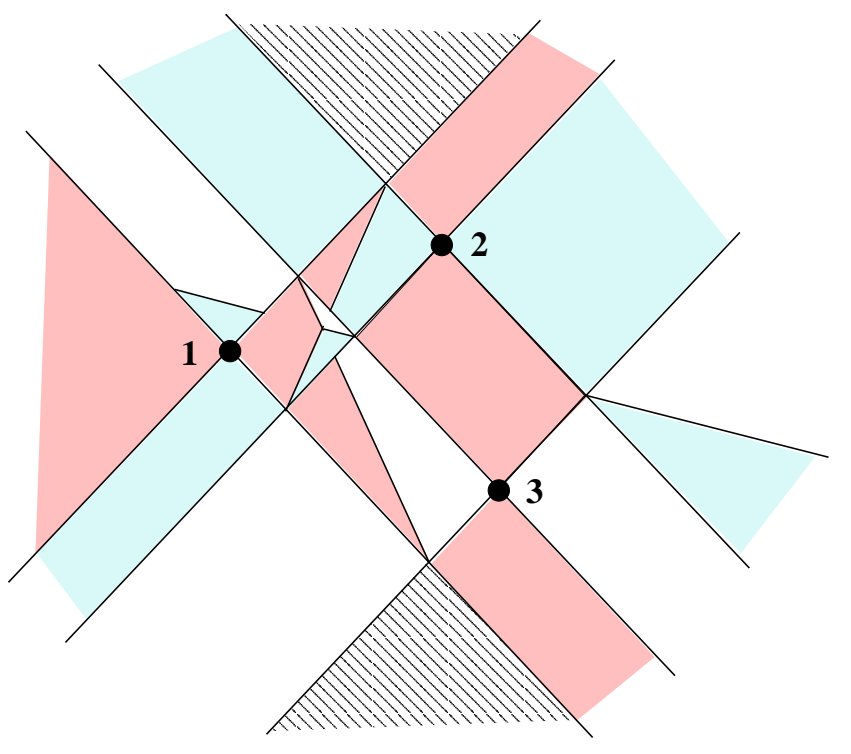

Figure 5. Distance $\Delta_{\infty}$

be contained in $\operatorname{reg}\left(\sigma_{i}\right)$, as 0 is the smallest value achieved by $\Delta_{\infty}$. This implies that the double-cones show up completely in the diagram; in fact, the diagram is a refinement of the arrangement induced in $R^{k}$ by these $n$ double-cones. Thus its size is $\Omega\left(n^{2}\right)$ already in $R^{2}$.

An example is given in Figure 5. For each sphere, its index is placed in its region. The hatched area is at distance $\infty$ from all three spheres. It is the intersection of the interiors of all double-cones.

As being the case for variant 1 , diagram vertices do arise which are not vertices of the double-cone arrangement. The situation is, however, more complicated for the present variant. Let $Z$ be a cell of this arrangement. Then, in the Voronoi diagram for $\Delta_{\infty}$, cell $Z$ is divided among the $m \leq n$ spheres $\sigma_{j}$ where $Z$ is exterior to cone $\left(\sigma_{j}\right)$. Division takes place with respect to the quasi-euclidean distance $d$ (which is non-negative in the entire cell). That is, $Z$ houses a part of the power diagram for $m$ spheres. In $R^{2}$, this implies an upper bound of $O\left(n^{3}\right)$ on the size of the diagram: There are $\Theta\left(n^{2}\right)$ arrangement cells, and each of them contains $O(n)$ (parts of) regions and edges of a power diagram.

We do not consider the $O\left(n^{3}\right)$ bound above to be asymptotically tight, but rather conjecture that $\Theta\left(n^{2}\right)$ is the true answer in $R^{2}$. There are two facts that complicate the combinatorial analysis of the diagram. First, the induced complex is not face-to-face. Second, and as a related fact, a sphere may occupy with its power region part of a cell $Z$ without contributing to the boundary of $Z$ with its doublecone. In Figure 5, this happens for sphere $\sigma_{2}$ and the cell in the upper left corner. For $R^{3}$, an obvious lower bound is $\Omega\left(n^{3}\right)$.

\section{Variant 3}

In order to consider the other extreme situation, we define the distance

$$
\Delta_{0}(p, q)= \begin{cases}d(p, q) & \text { if }>0 \\ 0 & \text { otherwise }\end{cases}
$$

Now negative squared tangent length means being as close as possible. Consequently, the diagram lives in the complement, $C$, of the union of the interiors of the respective double-cones. That is, lifetime of particles is irrelevant again, apart from the fact that it restricts the diagram to $C$. Note that $C$ consists of two or more maximal interiorconnected components; see Figure 6.

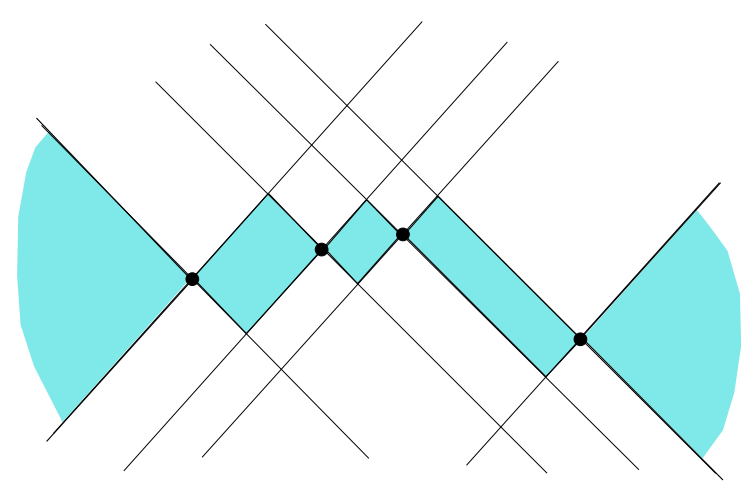

Figure 6. Active region $C$ for $\Delta_{0}$

For each point $x$ in the interior of $C$, the tangent length to all spheres $\sigma_{i}$ is positive. Within $C$, the diagram coincides with the standard case, i.e., the power diagram from Section 2. Notice that the induced cell complex is face-to-face: Any point on the boundary of $C$ that lies in the intersection of two double-cones also lies on the separator of the respective two spheres.

Incident to the boundary of $C$ but exterior, there are cells of the double-cone arrangement where the distance to exactly one sphere $\sigma_{i}$ is zero. These cells are, therefore, part of $\operatorname{reg}\left(\sigma_{i}\right)$. We exclude such parts of regions from consideration, as they do not change characteristical diagram properties.

Figure 7 depicts the diagram in $R^{2}$ for five spheres. Again, encirculated indices attach regions to their defining spheres. Parts of regions not contained in $C$ are lightly shaded. Their index is identical to that of the adjacent part. The region of sphere $\sigma_{2}$ is empty.

In $R^{2}$, the boundary of $C$ consists of $O(n)$ line segments. Diagram vertices are either vertices of $C$ (distance zero), or vertices of the standard power diagram. Hence their number is $O(n)$. The complex induced in $C$ is polygonal. After 
having computed the boundary of $C$, as well as the power diagram, in $O(n \log n)$ time, the parts of this power diagram within $C$ can be singled out in $O(n)$ time using the edgeto-edge property of the complex.

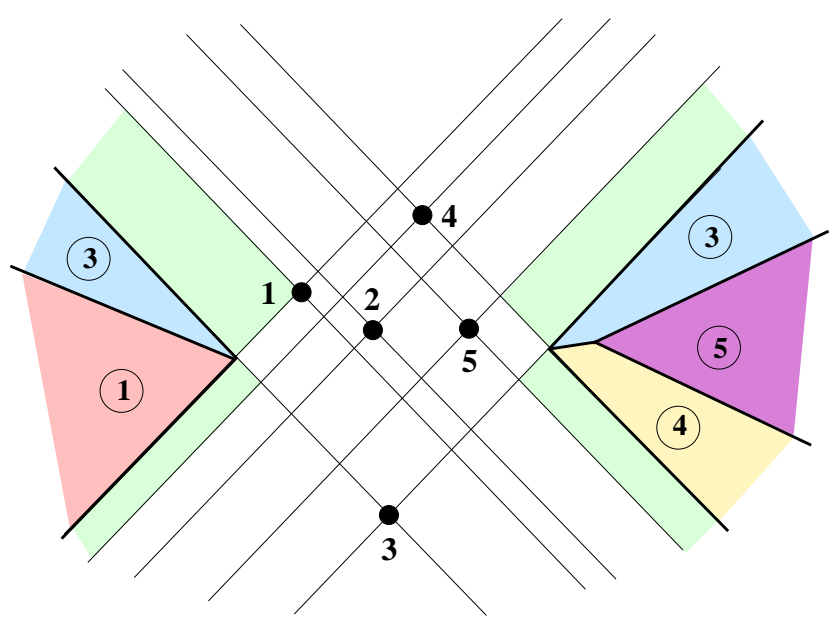

Figure 7. Distance $\Delta_{0}$

The situation remains relatively simple in $R^{3}$. To compute the boundary of $C$, we first treat the lower parts and the upper parts of the interiors of the double-cones separately and calculate their respective unions. As all cones are vertical and have the same angle of aperture, this task is equivalent to (twice) computing an additively weighted Voronoi diagram in $R^{2}$; see e.g. $[1,2]$. Such a diagram can be computed in $O(n \log n)$ time [7]. In a next step, the standard power diagram in $R^{3}$ is constructed in time $O\left(n^{2}\right)$. Exploiting, again, the face-to-face property, we can find the parts of the power diagram lying on the appropriate sides of the cone unions' boundaries. Observe that the combinatorial complexity of the obtained Voronoi diagram for distance $\Delta_{0}$ in $R^{3}$ is $O\left(n^{2}\right)$, as each boundary component of $C$ injectively corresponds to a face of the power diagram.

\section{Discussion}

We have considered several variants of decomposing space based on closeness with respect to the quasi-euclidean distance $d$. The resulting Voronoi diagrams exhibit quite different behavior concerning combinatorial size as well as geometric shape. Variants 1 and 2 behave quadratically in size already in $R^{2}$, and curved region boundaries add to the geometric complexity of these diagrams in $R^{3}$. Variant 3 , on the other hand, is (essentially) composed of linear components well treatable even in $R^{3}$. We raise the questions of whether there are other meaningful variants based on the distance $d$.
A related interesting question is whether one can define a meaningful concept of Delaunay triangulation for (variants of) the quasi-euclidean distance $d$ in $R^{2}$. A circle for $d$ with center $p$ and radius $r$ may be defined as $\left\{x \in R^{2} \mid d(x, p)=r\right\}$. This describes an equilateral hyperbola with center $p$. Using quasi-euclidean circumcircles of a triangles, combined with the empty circle property of a Delaunay triangulation, seems to be a possible approach.

Our results also substantiate the role power diagrams play in physical contexts. For all the variants discussed, the resulting Voronoi diagrams are, at least partially, composed of power diagrams. Another recent example where power diagrams relate to physics are the Bregman Voronoi diagrams considered in [9].

\section{References}

[1] F. Aurenhammer. Power diagrams: properties, algorithms, and applications. SIAM J. Computing 16 (1987), 78-96.

[2] F. Aurenhammer, H. Imai. Geometric relations among Voronoi diagrams. Geometriae Dedicata 27 (1988), 65-75.

[3] W. Benz. Classical Geometries in Modern Contexts; Geometry of Real Inner Product Spaces. Birkhäuser, Basel, Boston, Berlin, 2005.

[4] J.-D. Boissonnat, M. Teillaud. Effective Computational Geometry for Curves and Surfaces. Springer Berlin, Heidelberg, 2007.

[5] B. Chazelle, R.L.S. Drysdale, D.T. Lee. Computing the largest empty rectangle. SIAM J. Computing 15 (1986), 300-315.

[6] H. Edelsbrunner, R. Seidel. Voronoi diagrams and arrangements. Discrete \& Computational Geometry 1 (1986), 25-44.

[7] S. Fortune. A sweepline algorithm for Voronoi diagrams. Algorithmica 2 (1987), 153-174.

[8] H. Imai, M. Iri, K. Murota. Voronoi diagram in the Laguerre geometry and its applications. SIAM J. Computing 14 (1985), 93-105.

[9] F. Nielsen, J-D. Boissonnat, R. Nock. On Bregman Voronoi diagrams. ACM-SIAM Symp. on Discrete Algorithms, SODA, 2007.

[10] N.M.J. Woodhouse. Special Relativity. Springer Undergraduate Mathematics Series, 2003. 